Catcheside, D. G. (1954). J. gen. Microbiol. 11, 34-36.

\title{
Isolation of Nutritional Mutants of Neurospora crassa by Filtration Enrichment
}

\author{
By D. G. CATCHESIDE \\ Department of Genetics, University of Adelaide, South Australia
}

SUMMARY : Nutritional mutants of Neurospora crassa may be isolated by incubation of microconidia in a liquid medium deficient in specific growth factors followed by filtration and then growth on supplemented media of the conidia remaining in the filtrate.

A method for the isolation of nutritional mutants of Neurospora crassa by filtration and subsequent selective enrichment of the medium has been developed as an adaptation of the one devised by Fries (1947) for Ophiostoma multiannulatum. It involves the use of a strain which produces microconidia abundantly, and preferably a strain that will grow as a colony or respond well by growing as a colonial paramorph on a suitable medium (Tatum, Barratt \& Cutter, 1949). The significance of the use of microconidial strains is that only in a microconidium will a mutant nucleus have a chance of growing ultimately into a colony with the mutant character. A macroconidium containing a mutant nucleus would germinate and grow on minimal medium by virtue of the presence of one or more non-mutant nuclei, and so would be discarded in the course of the operations designed to eliminate as many as possible of the conidia which contain non-mutant nuclei. Stocks of $N$. crassa such as $\mathrm{Y} \mathrm{8743,} \mathrm{which} \mathrm{is} \mathrm{colonial} \mathrm{and} \mathrm{peach-microconidial} \mathrm{(Barratt} \mathrm{\&} \mathrm{Garnjobst,}$ 1949), or the peach fluffy double mutant, which is not colonial, are suitable. They should be grown on a medium which induces the production of a large number of conidia. Some strains produce microconidia almost exclusively if the inoculum is buried under a layer of agar. The mycelium grows through this and produces a dense crop of microconidia on the surface.

The microconidia are harvested by washing them off the surface of the culture with sterile distilled water. The suspension is then filtered by means of a sterile cotton-wool filter to remove fragments of mycelium, yielding a dense opalescent suspension of microconidia. The filter is made by blowing a hole in the bottom of a Pyrex or other hard glass test-tube, and fusing to this hole a short length of Pyrex tube of about $7 \mathrm{~mm}$. diameter to form a drainage stem. A small piece of absorbent cotton-wool is inserted into the base of the filter and just within the drainage stem. The open end of the filter is plugged with non-absorbent cotton-wool and a wrapping of this is also made round the stem and inserted into an intact test-tube. The whole assembly is then autoclaved.

The filtered suspension is irradiated with ultraviolet light by either of two methods: (1) the suspension is transferred to a sterile Petri dish and rocked 
with the cover off under a suitable ultraviolet lamp; (2) the suspension is transferred to a sterile quartz tube which is then sealed with a sterile cork so that not less than one-eighth of the volume is occupied by air; the tube is clamped in a suitable arrangement so that it is held horizontally and may be rotated continuously about this axis under a suitable ultraviolet lamp. The exposure time must be found for a given apparatus by trial.

The lamp used was a low pressure mercury vapour burner T/M5/369, obtained from the Thermal Syndicate Ltd., Vitreosil Works, Wallsend-onTyne. Two minute exposure at a distance of $15 \mathrm{~cm}$. was used for Neurospora crassa microconidia, $30 \mathrm{sec}$. for haploid yeast and $8 \mathrm{~min}$. for Chromocrea spinulosa ascospores.

After irradiation, samples of the conidial suspension are transferred to $100 \mathrm{ml}$. Erlenmeyer flasks containing $20 \mathrm{ml}$. liquid minimal medium. Westergaard \& Mitchell's formula (1947) is a suitable one, but may have to be modified if the fungus grows too vigorously. The substitution of sorbose for some of the glucose or sucrose helps to decrease the growth rate, but the optimal proportions must be found empirically for a given stock. Commonly, about $0.8 \%$ sorbose, together with $1 \%$ sucrose, gives a convenient diminution of growth rate. However, a lesser proportion of sucrose may be needed in the plating medium referred to later.

The flasks are placed in a shaking machine and agitated continuously while being incubated at $25^{\circ}$ or at a higher temperature when temperature-sensitive mutants are sought. Agitation should be fairly gentle, the flasks being moved in each direction about once a second. Too violent agitation may throw liquid on to the plug where the fungus may grow and conidiate and so contaminate the suspension with further unwanted conidia. After not more than $24 \mathrm{hr}$. the contents of each flask are filtered through a sterile cotton-wool filter into a fresh sterile Erlenmeyer flask.

During the period of incubation many of those conidia which are capable of growth in the minimal medium form small spherical colonies. These colonies do not pass through the cotton-wool filter but the great majority of the ungerminated conidia do. The flasks containing the filtrate are returned to the shaker and incubated for further periods, each followed by filtration, the intervals between successive filtrations being adjusted according to the rapidity and amount of growth, until no more visible growth occurs in the flasks. This normally requires 3 or even 4 days of incubation, since after irradiation a number of conidia, ultimately capable of germinating in minimal medium, are delayed, sometimes for more than $\mathbf{2}$ days.

When little or no further growth appears, samples of the remaining suspension, after a final filtration, are plated in Petri dishes containing agar medium of composition similar to that employed in the flasks, but supplemented with the factor or factors for which nutritionally deficient mutants are sought. When colonies have grown after incubation, transfers are made to supplemented slopes and the isolates are tested individually. The usual experience is that a number of the isolates are non-mutants, delayed in germination, but an appreciable proportion of the isolates are of the kind desired. 
Outcrosses and re-isolation are necessary to rid the stocks of undesirable characters, such as colonial growth and microconidial habit, which were essential in the stock to provide uninucleate conidia.

Table 1 summarizes some of the experiences in the use of the filtration enrichment method with Neurospora crassa stock Y 8743. The first line of the Table illustrates the very low yield of mutants obtained when no selective enrichment is practised, but instead the irradiated conidia are plated out in suitable dilution and every colony tested individually.

Table 1. Yields of nutritional mutants from total isolation and filtration enrichment

\begin{tabular}{llcrc}
\multicolumn{1}{c}{$\begin{array}{l}\text { Method } \\
\text { Total isolation }\end{array}$} & $\begin{array}{l}\text { Supplement used } \\
\text { Malt extract }\end{array}$ & $\begin{array}{c}\text { No. of } \\
\text { expts. }\end{array}$ & $\begin{array}{c}\text { Total } \\
\text { isolated }\end{array}$ & $\begin{array}{c}\text { Mutants } \\
\text { obtained }\end{array}$ \\
& Malt extract & 10 & 1691 & 9 \\
Filtration enrichment & Histidine & 4 & 273 & 39 \\
& Ammonium salt & 1 & 43 & 3 \\
& Casein hydrolysate & 1 & 41 & 4 \\
& Adenine & 1 & 31 & 4 \\
Proline & 1 & 44 & 3 \\
Methionine & 1 & 47 & 2 \\
& & & 105 & 3
\end{tabular}

The filtration enrichment method works most satisfactorily for mutants which require a considerable amount of growth factor to achieve appreciable growth. For those mutants which require a relatively very small amount of supplement, such as those which require a vitamin, centrifugation and washing to remove growth factors liberated during growth or by conidia killed during the irradiation may be essential to success. The method is adaptable to a number of particular problems by suitable modification of the medium used during the shaking process.

\section{REFERENCES}

Barratt, R. W. \& Garnjobst, L. (1949). Genetics of a colonial microconidial strain of Neurospora crassa. Genetics, 34, 351.

Fries, N. (1947). Experiments with different methods of isolating physiological mutations of filamentous fungi. Nature, Lond. 159, 199.

Tatum, E. L., Barratt, R. W. \& Cutter, V. M. (1949). Chemical induction of colonial paramorphs in Neurospora and Syncephalastrum. Science, 109, 509.

WestergaArd, M. \& Mitchell, H. K. (1947). Neurospora, V. A synthetic medium favouring sexual reproduction. Amer. J. Bot. 34, 573.

(Received 22 December 1953) 